\title{
Outcome of treatment of vulvodynia by local application of anaesthetic agent
}

\section{Pratiksha Gupta*, Chandana Shekhar}

Department of Obstetrics \& Gynaecology, PGIMSR, ESI, Basaidarapur, New Delhi

Received: 10 February 2016

Accepted: 05 March 2016

\section{*Correspondence:}

Dr. Pratiksha Gupta,

E-mail: drpratiksha@gmail.com

Copyright: (c) the author(s), publisher and licensee Medip Academy. This is an open-access article distributed under the terms of the Creative Commons Attribution Non-Commercial License, which permits unrestricted non-commercial use, distribution, and reproduction in any medium, provided the original work is properly cited.

\begin{abstract}
Background: Vulvodynia is a chronic gynecological disorder. It has a widespread effect on quality of life due to physical disabilities, psychological distress and sexual dysfunction. The objective of the study was to assess the effectiveness of application of 5\% Xylocaine ointment for treatment of Vulvodynia.

Methods: This prospective study was conducted in the department of Obstetrics and Gynecology in Government Medical College and Hospital Chandigarh, from November 2004 to October 2010. Study included 520 female patients (age range, 20-65 years; mean, 32.50 years) with the diagnosis of Vulvodynia. Vulvodynia was measured by using Friedrich criteria. Daily and intercourse related pain was measured using a 10-mm visual analogue scale. Comparison was done between ability to have intercourse and pain ratings before and after the treatment.

Results: After a mean of 8 weeks' treatment, the daily pain rating was 10.37 points lower as compared to pretreatment phase. The intercourse-related pain score was 39.11 points lower after treatment. After six months of follow-up, eighty-eight percent (458/520) of women reported ability to have intercourse after treatment compared with $34 \% 177 / 520$ ) at the initial visit. Visual analogue score of 520 patients were significantly reduced from 8.6 to 2.5 after post treatment.

Conclusions: Topical 5\% Xylocaine ointment was associated with an improvement in symptoms in the majority of women with Vulvodynia.
\end{abstract}

Keywords: Vulvodynia, Women, Vaginal mucosa, 5\% Xylocaine

\section{INTRODUCTION}

Vulvodynia syndrome is a chronic, heterogeneous, and multifactorial Gynecological condition with an estimated prevalence of $9-12 \%$, between $20-50$ years of age. ${ }^{3}$ It has a widespread effect on quality of life due to physical disabilities, psychological distress and sexual dysfunction. ${ }^{1,2}$ Fifteen percent of women seen in the outpatient departments have reported pain on palpating areas of the Vulvar vestibule. The current and most widely accepted classification of Vulvar pain as devised by the International Society for the Study of Vulvar Disease (ISSVD) classified Vulvar pain related to a specific disorder (infections, inflammatory, neoplastic or neurologic) and Vulvodynia (pain due to nonspecific etiology) Vulvodynia not only causes pain, but often leads to sexual dysfunction, including arousal and orgasmic difficulty. ${ }^{4-6}$ Patients may feel burning, stinging, rawness, aching, soreness, or throbbing that occurs in the vulva, labia, or vagina. There is no single treatment regimen or combination of treatments which have been shown to consistently improve the symptoms. A large proportion of patients attend multiple medical consultations before receiving a diagnosis, and in this study, we performed a prospective analysis of the clinical characteristics and the outcomes of application of $5 \%$ Xylocaine ointment in the treatment of patients with Vulvodynia. 


\section{METHODS}

This prospective study was conducted in the department of Obstetrics and Gynaecology in Government Medical College and Hospital Chandigarh, from November 2004 to October 2010. It was approved by institutional medical ethics committee. It included 520 female patients (age range, 20-65 years; mean, 32.50 years) with the diagnosis on Vulvodynia. A written informed consent was obtained from all the patients. All patients were evaluated for Vulvar pain by taking a standardized history and physical examination. We used 10-mm visual analogue scale for discomfort level during daily activity and attempted intercourse. We performed comprehensive physical examinations for all women using a standardized form to record findings. Cotton swab stick was used to perform the test. cotton swab was touched to vestibule, labia majora and labia minora, and recording of the presence or absence of allodynia, sensory loss, and the degree of erythema, the higher the score, the more severe the symptoms. Vulvodynia was diagnosed using Friedrich criteria (Table 1). ${ }^{7}$

Patients were instructed to apply a copious amount of 5\% Xylocaine ointment to the affected area at bedtime and were asked to place a cotton ball generously coated with the 5\% Xylocaine ointment in the vestibule to assure continuing overnight application. We instructed patients to use the treatment overnight, for 8 or more hours. We obtained repeat visual analogue scale scores for pain with daily activity and pain with intercourse at the follow-up visit scheduled 6-8 weeks after initiation of treatment. Patients were also asked about ability to have intercourse at both the initial and the follow-up visit. Patients were defined as able to have intercourse if they reported any intercourse during a given month, regardless of frequency or degree of discomfort. Those who were celibate because of severity of symptoms were defined as unable to have intercourse. Regardless of the patients' compliance with treatment, or subsequent additional diagnoses, the data for all the patients who met criteria, were given treatment, and were kept in follow up for 6 months. The primary outcome was a change in daily and intercourse-related visual analogue scale scores with treatment. Because sexual dysfunction is the primary concern for patients, we also assessed patients' selfreported ability to have intercourse.

\section{RESULTS}

Five hundred twenty patients with Vulvodynia were included in the study group. This study, 66\% (343/520) women at initial questionnaire were unable to have sexual intercourse due to pain. The mean age of women was 32.50 years (20-65 years of age). Of the 520 women, 110 $(21.15 \%)$ were illiterate, $105(20.19 \%)$ had primary school education, $120(23.07 \%)$ had up to secondary school, 95 (18.26\%) up to graduation and $90(17.30 \%)$ up to post graduation. Pelvic examination was done for every woman, and vaginal samples were taken at the same time. Vulvodynia was by identifying vaginal $\mathrm{pH}$, smear preparing and staining, and culturing. Pretreatment visual analogue score of 520 patients were 8.6 (range, 6-10). Most women had tried other treatments, including topical medications (estrogen cream, metronidazole gel, antifungal cream, corticosteroid cream, and trichloroacetic acid), amitriptyline, oral herbal remedies, and revision of episiotomy. Topical 5\% Xylocaine ointment was associated with an improvement in symptoms in the majority of women. After a mean of 8 weeks' treatment, the daily pain rating was 10.37 points lower than in pre-treatment. The intercourse-related pain score was 39.11 points lower after treatment. After six months of follow-up, eighty-eight percent (458/520) of women reported ability to have intercourse after treatment compared with $34 \%$ 177/520) at the initial visit. Post treatment visual analogue score of 520 patients were significantly reduced to 2.5 (range, $0-5$ ). After six months of follow-up, $88 \%$ (458/520) reported on-going use of $5 \%$ Xylocaine ointment. $8.65 \%(45 / 520)$ patients were using it infrequently, several times a week, weekly or less. There was no complication of local Xylocaine application. The results were excellent in $88 \%$ (458/520) and good in $8.65 \%(45 / 520)$ patients. At 6 months, subjective overall assessment was done, $88 \%$ (458/520), patients were fully satisfied, and $8.65 \%$ (45/520) were satisfied with the outcome of treatment.

Table 1: Friedrich criteria for vulvodynia diagnosis.

\begin{tabular}{|llllll|}
\hline Score & Dyspareunia & burning & Itching & Swab test & Erythema \\
\hline 0 (Absent) & Absent & Absent & Absent & Negative & Absent \\
\hline 1 (Mild) & Mild pain & Mild pain & Mild pain & Weakly positive & Mild pain \\
\hline 2 (Moderate) & Persistent & Moderate & Moderate & positive & Moderate \\
\hline 3 (Severe) & With intercourse & Severe & Severe & Strongly positive & Severe \\
\hline
\end{tabular}

\section{DISCUSSION}

There is varied treatment regimens used for the treatment of Vulvodynia. Initial medical approaches include the avoidance of all skin and mucosal irritants (like soap, perfumes, and deodorants), a diet containing low oxalates, scarce simple carbohydrates, and oral supplementation of calcium citrate. Additional measures are wearing cotton underwear, a trial of diverse relaxation techniques and psychological assessment with counseling 
and group support. ${ }^{8}$ Topical treatments that have been reported as beneficial in multiple studies include topical gel anesthetics and estrogen cream particularly in perimenopausal women. Regional therapies including pudendal nerve block and pelvic floor muscle rehabilitation have also been recommended. ${ }^{9,10}$ Physical therapy has also shown to be effective in the treatment of Vulvodynia. ${ }^{11}$ It involves the evaluation of the patient pelvic musculature, joints, fascia and tendons. Exercise for the pelvic floor and girdle, soft tissue mobilization, joint manipulation, muscle relaxation and general tone balance are also included in treatment plan. However, standardization regarding the effective treatment approach is inexistent among therapists therefore outcomes cannot be accurately validated nor reproduced. ${ }^{12}$ Because histological features of the Vulvar epithelium can show an increased density of neural tissue and nonspecific inflammation, neuromodulators have been used. Oral medications include tricyclic antidepressants like amitriptiline, nortriptiline, and anticonvulsants like gabapentin. Opiates should be utilized only for short periods in acute settings and are not recommended as maintenance therapy. ${ }^{13}$ Surgical excision of the Vulvar tissue involved should be considered in unresponsive cases. ${ }^{14,15}$ Success from vestibulectomy varies between 65 and 90 percent but long-term relief is uncertain. ${ }^{16,17}$ Laser ablation of the Vulvar epithelium with the KTP-Nd: YAG Laser and the $\mathrm{CO}_{2}$ Laser, has been promoted as an option to the more invasive vestibulectomy. Cognitive Behavior Therapy (CBT) has been applied to women with Vulvodynia with encouraging results. ${ }^{18}$ other methods of treatment such as acupuncture, local treatment using casaicin, ketoconazole, estrogen, steroids, niterfron, nifedipine and hypnosis are used to a lesser extent and with mixed results although without any major study reported. Most women treated in our study - with topical Xylocaine 5\% ointment, noted improvement in symptoms. The majority had tried other medications without relief. This treatment is simple, safe, and inexpensive. ${ }^{19}$ In our study, After six months of follow-up, eighty-eight percent (458/520) of women reported ability to have intercourse after treatment compared with $34 \%$ 177/520) at the initial visit.

Histologic studies show a proliferation of C-fibers (which carry pain sensation) and the presence of calcitonin generelated peptide (commonly seen with nerve irritation) in Vulvar vestibulitis patients. In addition, patients with Vulvar vestibulitis have abnormalities of sensory perception, such as heat and touch. ${ }^{8}$ Xylocaine blocks transmission of C-fibers. Continuous exposure to Xylocaine inhibits irritable nociceptors and is the proposed mechanism by which it provides benefit in treatment of chronic pain.

Due to slow onset and moderate duration of action of xylocaine, makes it a potentially useful treatment for Vulvar vestibulitis. Topical and injected local anaesthetics have been found effective in treating postherpetic neuralgia. ${ }^{20}$ Some patients using topical anaesthetics attain sustained relief, whereas others many need to continue treatment for many years to maintain pain relief. In our study, Xylocaine has been the only beneficial treatment in women with severe symptoms. In our study, topical 5\% Xylocaine ointment was associated with an improvement in symptoms in $88 \%$ of women. A woman with severe allodynia over the anterior vestibule does not respond to the treatment well. Those with prior surgery responded favourably to the treatment as long as the area of allodynia and tenderness was limited to the posterior vestibule. In present study, at 6 months of follow up, subjective overall assessment was done which showed that $88 \%(458 / 520)$ patients were fully satisfied, and $8.65 \%(45 / 520)$ were satisfied with the outcome of treatment. Present study concluded that overnight topical Xylocaine therapy improves symptoms in women with refractory Vulvar vestibulitis.

\section{CONCLUSIONS}

Topical 5\% Xylocaine's slow onset of action and moderate duration makes it a potentially useful treatment for chronic pain disorders such Vulvar vestibulitis, but based on the present study more randomised control trail is required.

\section{Funding: Funds of GMCH \\ Conflict of interest: None declared}

Ethical approval: The study was approved by the Institutional Ethics Committee

\section{REFERENCES}

1. Petersen CD, Lundvall L, Kristensen E, Giraldi A. Vulvodynia. Definition, diagnosis and treatment. Acta Obstet Gynecol Scand. 2008;87(9):893-901.

2. Arnold LD, Bachmann GA, Rosen R, Kelly S, Rhoads GG. Vulvodynia: characteristics and associations with comorbidities and quality of life. Obstet Gynecol. 2006;107(3):617-24.

3. Hengge UR, Runnebaum IB. Vulvodynia. Hautarzt 2005;56(6):556-9.

4. Reed BD, Haefner HK, Edwards L. A survey on diagnosis and treatment of Vulvodynia among Vulvodynia researchers and members of the International Society for the Study of Vulvovaginal Disease. J Reprod Med. 2008;53(12):921-9.

5. Lotery HE, McClure N, Galask RP. Vulvodynia. Lancet. 2004;363:1058-60.

6. Yoon H, Chung WS, Shim BS. Botulinum toxin A for the management of Vulvodynia. Int J Impot Res. 2007;19:84-7.

7. Friedrich EG Jr. Vulvar vestibulitis syndrome. J Reprod Med. 1987;32:110-4.

8. Masheb RM, Kerns RD, Lozano C, Minkin MJ, Richman S. A randomized clinical trial for women with Vulvodynia: Cognitive-behavioral therapy vs. supportive psychotherapy. Pain. 2009;141(1-2):3140 . 
9. Hartmann D, Strauhal MJ, Nelson CA. Treatment of women in the United States with localized, provoked Vulvodynia: practice survey of women's health physical therapists. J Reprod Med. 2007;52(1):48-52.

10. Murina F, Bernorio R, Palmiotto R. The use of amielle vaginal trainers as adjuvant in the treatment of vestibulodynia: an observational multicentric study. Medscape J Med. 2008;10(1):23.

11. Backman H, Widenbrant M, Bohm-Starke N, Dahlof LG. Combined physical and psychosexual therapy for provoked vestibulodynia-an evaluation of a multidisciplinary treatment model. J Sex Res. 2008;45(4):378-85.

12. McCormack WM, Spence MR. Evaluation of the surgical treatment of Vulvar vestibulitis. Eur J Obstet Gynecol Reprod Biol. 1999;86(2):135-8.

13. Bergeron S, Bouchard C, Fortier M, Binik YM, Khalife S. The surgical treatment of Vulvar vestibulitis syndrome: a follow-up study. J Sex Marital Ther. 1997;23(4):317-25.

14. Eva LJ, Narain S, Orakwue CO, Luesley DM. Is modified vestibulectomy for localized provoked Vulvodynia an effective long-term treatment? A follow-up study. J Reprod Med. 2008;53(6):435-40.

15. Leclair CM, Goetsch MF, Lee KK, Jensen JT. KTPnd: YAG laser therapy for the treatment of vestibulodynia: a follow-up study. J Reprod Med. 2007;52(1):53-8.

16. Reed BD, Haefner HK, Sen A, Gorenflo DW. Vulvodynia incidence and remission rates among adult women: a 2-year follow-up study. Obstet Gynecol. 2008;112:231-7.

17. Bergeron S, Binik YM, Khalife S, Pagidas K. Vulvar vestibulitis syndrome: a critical review. Clin J Pain. 1997;13(1):27-42.

18. Ehrstrom S, Kornfeld D, Rylander E, Bohm-Starke N. Chronic stress in women with localised provoked Vulvodynia. J Psychosom Obstet Gynaecol. 2009;30(1):73-9.

19. Reese JB. Results from an RCT testing a psychosocial treatment for Vulvodynia: Methodological strengths and future directions. Pain. 2009;141(1-2):8-9.

20. Rowbotham MC, Fields HL. Topical Xylocaine reduces pain in post-herpetic neuralgia. Pain. 1989;38:297-301.

Cite this article as: Gupta $\mathrm{P}$, Shekhar C. Outcome of treatment of vulvodynia by local application of anaesthetic agent. Int J Reprod Contracept Obstet Gynecol 2016;5:1167-70. 